\title{
DEL CONGRESO A LOS SUBURBIOS: INICIATIVAS LOCALES PARA EL CONTROL DE LA MIGRACIÓN EN ESTADOS UNIDOS
}

\author{
ALEXANDRA DÉLANO ALONSO*
}

RESUMEN: Este artículo analiza las implicaciones de las iniciativas de ley y ordenanzas locales para controlar la migración que se han propuesto en numerosos estados y ciudades de Estados Unidos ante el fracaso de la reforma migratoria en el Congreso. La primera parte describe las facultades de las autoridades locales y estatales para garantizar el cumplimiento de las leyes migratorias, así como los cambios en la legislación e instrumentación de estas medidas a partir del once de septiembre. La segunda parte examina casos recientes de ordenanzas e iniciativas de ley propuestas por gobiernos estatales y asambleas locales para enfrentar el crecimiento de la población de inmigrantes indocumentados. Finalmente, propongo una reflexión sobre la relevancia de esta situación en el contexto del debate más amplio sobre la reforma migratoria en Estados Unidos, los retos que representa para el gobierno mexicano y la posibilidad de aprovechar la labor de varios actores que participan en el debate a nivel local promoviendo medidas a favor de los inmigrantes frente a esta ola anti-inmigrante.

PAlABRAS ClAVE: Migración México-Estados Unidos; política migratoria; reforma migratoria; migración indocumentada; ordenanzas; jornaleros.

ABSTRACT: This paper analyzes the implications of local ordinances and law initiatives aimed at controlling immigration, which have been proposed in various states and cities in the United States in the context of the failure of immigration reform in Congress. The first part describes local and state officials' responsibilities in terms of immigration law enforcement, as well as the changes in the legislation and the implementation of these measures after $9 / 11$. The second part examines recent cases of local ordinances and law initiatives proposed by state assemblies and local councils in response to the growing population of undocumented immigrants. Finally, I consider the relevance of this situation in relation to the broader debate on immigration reform in the US, the challenges it represents for the Mexican government and the possibilities that it offers in terms of working together with

\footnotetext{
* Department of Politics and International Relations, University of Oxford.
} 
various actors at the local level who actively promote measures in favor of immigrants' rights and against this anti-immigrant wave.

KEYWORDS: Mexico-US migration; migration policies; immigration reform; undocumented migration; local ordinances; day laborers.

\section{INTRODUCCIÓN}

partir del once de septiembre de 2001, el debate migratorio en Estados
Unidos se ha concentrado principalmente en los aspectos de seguri-
dad, mientras que las medidas relacionadas con el tema se han dirigi-
do al reforzamiento del control de las fronteras. Este enfoque tiene consecuencias negativas para la población inmigrante en ese país (independientemente de su estatus migratorio) porque conlleva el endurecimiento de los argumentos «anti-inmigrantes» de los grupos que asocian a la migración con el terrorismo, el crimen, la violencia y la pérdida de los «valores» norteamericanos. Implica, además, mayores riesgos para quienes siguen cruzando la frontera por vías peligrosas y aumenta las dificultades para que los inmigrantes residentes en Estados Unidos - principalmente aquellos con estatus irregular- desarrollen sus actividades sin ser objeto de violaciones a sus derechos humanos.

A la par de este proceso, se ha desarrollado otra tendencia igualmente preocupante en términos de los efectos negativos que tiene sobre la situación de los inmigrantes de origen latino/hispano. Desde 2001, los gobiernos locales y estatales en Estados Unidos han decidido tomar acciones propias para controlar la migración indocumentada, lo cual refleja las presiones de grupos dentro de la sociedad norteamericana que se sienten «amenazados» por el reciente aumento de flujos de inmigrantes dentro de localidades suburbanas en las que tradicionalmente no existían estas minorías, o eran mucho más pequeñas. Ante estas circunstancias, organizaciones y grupos civiles que trabajan para apoyar y defender a los inmigrantes indocumentados han tomado acciones para oponerse decididamente a estas medidas. En varios casos han tenido un éxito inesperado que sienta precedentes importantes en el debate y la legislación sobre este tema.

Esta situación es ilustrativa respecto a las divisiones que históricamente han existido dentro de la sociedad y el gobierno norteamericano sobre los costos y beneficios de la migración y la manera en que se deben administrar estos flujos. Asimismo, reflejan la frustración que prevalece en el ámbito local sobre el fracaso de las políticas migratorias existentes, principalmente en el contexto del aumento de la migración a zonas no tradicionales y el vínculo entre seguridad y migración después del once de septiembre. Finalmente, destaca la importancia que han adquirido los grupos y organizaciones no gubernamentales que trabajan a favor de los derechos de los inmigrantes y la influencia que pueden llegar a tener en este debate, así como en la legislación correspondiente. 
En la primera parte de este artículo describo las facultades de las autoridades locales y estatales para garantizar el cumplimiento de las leyes migratorias, así como los cambios en la legislación e instrumentación de estas medidas a partir del once de septiembre. En la segunda parte examino casos recientes de ordenanzas e iniciativas de ley propuestas por gobiernos estatales y asambleas locales para enfrentar el crecimiento de la población de inmigrantes indocumentados. Finalmente, propongo una reflexión sobre la relevancia de esta situación en el contexto del debate más amplio sobre la reforma migratoria en Estados Unidos, los retos que representa para el gobierno mexicano y las posibilidades que ofrece en términos de aprovechar la labor de varios actores que participan en el debate a nivel local promoviendo medidas a favor de los inmigrantes frente a esta ola anti-inmigrante.

\section{FACULTADES MIGRATORIAS DE LOS GOBIERNOS LOCALES Y ESTATALES}

En Estados Unidos, el Congreso federal tiene la autoridad exclusiva para legislar en materia migratoria y garantizar el cumplimiento de las leyes correspondientes. Hasta 1996, la Ley de Inmigración y Naturalización (Immigration and Naturalization Act-INA) otorgaba a las autoridades locales y estatales estadounidenses la facultad de arrestar a las personas que cometieran algún delito relacionado con la ley migratoria (por ejemplo, tráfico de personas), pero no tenían autorización para detener a una persona ante la sospecha de violaciones civiles a la ley migratoria (por ejemplo, encontrarse en el país sin estatus legal), aunque podían proporcionar apoyo a las autoridades federales en estos casos. Al aprobarse la Ley Anti-Terrorismo y Pena de Muerte Efectiva (AEDPA) y la Ley de Reforma Migratoria y Responsabilidad Inmigrante (IIRIRA) en 1996, el Congreso estadounidense extendió las facultades de las autoridades locales y estatales para detener a inmigrantes que se encuentren en Estados Unidos de manera ilegal y que hayan sido acusados previamente de algún crimen dentro del país. Además, conforme a la IIRIRA, la policía estatal o local puede aplicar las leyes migratorias civiles en casos excepcionales. ${ }^{1}$ Así, conforme a estas leyes, las autoridades locales pueden ejercer ciertas funciones que corresponden al gobierno federal respecto de la aplicación de la ley migratoria, pero sólo en casos específicos y bajo ciertas condiciones.

La IIRIRA también permite a los estados y localidades establecer un acuerdo voluntario en forma de «Memorando de Entendimiento» con la Oficina de Migración y Aduanas (ICE), que da facultades a la policía local o estatal para garantizar el cumplimiento de la ley migratoria. El Memorando requiere que la policía obtenga un entrenamiento adecuado, establece candados específicos respecto a la apli-

\footnotetext{
${ }^{1}$ Estos casos incluirían las siguientes condiciones: cuando exista un «flujo masivo» de extranjeros conforme lo define la Ley INA, cuando la situación requiera una respuesta inmediata del gobierno federal y cuando los oficiales federales obtengan el consentimiento del departamento estatal o local encargado.
} 
cación de leyes migratorias civiles y obliga a las entidades locales a cubrir los gastos relacionados. Si un estado o localidad no quiere establecer este tipo de acuerdo, o tiene leyes que le impidan proceder con este tipo de aplicación de la ley migratoria (como sería el caso de los estados o ciudades «santuario»), no está obligado a participar, ni está sujeto a penalidades por no hacerlo. De acuerdo al reporte del National Immigration Forum, ${ }^{2}$ antes del 11 de septiembre de 2001, ninguna localidad había concretado una negociación para establecer el Memorando de Entendimiento, aunque al menos una ciudad, en este caso Salt Lake City, lo había intentado. ${ }^{3}$ No obstante la oposición de algunas autoridades, jefes de la policía, organizaciones de derechos civiles y representantes de grupos latinos, para junio de 2007 al menos 21 departamentos de policía en 11 estados habían establecido acuerdos con la Oficina de Migración y Aduanas para participar en actividades relacionadas con el cumplimiento de las leyes migratorias; cerca de 375 policías habían recibido el entrenamiento correspondiente y 71 solicitudes de acuerdo estaban pendientes. ${ }^{4}$

Finalmente, la IIRIRA estableció que los empleados del gobierno pueden reportar información sobre el estatus migratorio de otras personas a la oficina de Servicios de Naturalización e Inmigración (Immigration and Naturalization Services-INS -integrada desde 2003 al Departamento de Seguridad Interna-Department of Homeland Security -como la Oficina de Ciudadanía e Inmigración- US Citizenship and Immigration Services-USCIS), aunque no significa que tengan facultades para investigar el estatus migratorio de una persona, ni los obliga a utilizar esta información. La Ley de Responsabilidad Individual, Oportunidad en el Trabajo y Reconciliación (PRWORA), también aprobada en 1996, sí requiere a las agencias de bienestar social federales y locales reportar a inmigrantes indocumentados ante las autoridades migratorias.

Algunos estados y localidades tuvieron una respuesta negativa a estas leyes, bajo el argumento de que al poner en práctica este tipo de medidas, la población local perdería confianza en las autoridades y no reportaría incidentes ni daría testimonios por temor a que se cuestionara su estatus migratorio. Para prevenir esta situación, varias ciudades emitieron resoluciones que prohíben a la policía local aplicar las leyes migratorias y/o dan instrucciones a los servidores públicos de no negar servicios con base en criterios de estatus migratorio.

Después de los ataques terroristas del once de septiembre de 2001, el gobierno estadounidense se enfocó en el endurecimiento de las medidas de seguridad inter-

\footnotetext{
2 National Immigration Forum, «Backgrounder: Immigration Law Enforcement by State and Local Police», National Immigration Forum, mayo de 2004 (http://www.immigrationforum.org/Deskt opDefault.aspx?tabid=572, última consulta: 20 de noviembre de 2006).

${ }^{3}$ Al finalizar las negociaciones entre la ciudad de Salt Lake City y el Departamento de Justicia, el Memorando fue vetado por el Concejo de la Ciudad tras una audiencia con residentes de la localidad (véase National Immigration Forum, art. cit.).

${ }^{4}$ «Suburb seeking power to deport», Chicago Tribune, 20 de junio de 2007.
} 
nas para el control de la migración. El Departamento de Justicia solicitó el apoyo de las policías locales y estatales para fortalecer las medidas anti-terrorismo, incluyendo disposiciones para reforzar el cumplimiento de la ley migratoria, bajo el argumento de que el gobierno federal no tiene suficientes recursos ni personal para hacerlo. El Departamento enfrentó resistencia por parte de algunas policías locales y estatales en cuanto a entrevistar a personas bajo sospecha de tener vínculos con organizaciones terroristas, aunque no hubieran cometido ningún crimen. De acuerdo al National Immigration Forum, más de 20 ciudades y condados, así como 3 estados han adoptado políticas que limitan la colaboración entre la policía local y autoridades migratorias.

En 2002, el Procurador General anunció la creación de nuevos programas para controlar las entradas y salidas de extranjeros (National Security Entry-Exit Registration System (NSEERS) y National Crime Information Center (NCIC)) para lo cual requeriría la cooperación de las policías locales y estatales, otorgándoles la «autoridad inherente» para arrestar y detener a personas que hayan violado la ley migratoria y se encuentren en el registro del NCIC, lo cual implica que su autoridad se extiende de lo criminal a lo civil. Sin embargo, los criterios sobre las nuevas obligaciones de las policías locales y estatales no quedaron del todo claros. Algunas ciudades continuaron negociando los Memorandos de Entendimiento para ejercer estas facultades a pesar de que las nuevas medidas estipulaban que no sería necesario.

Al crearse el Departamento de Seguridad Interna a finales de 2002, se establecieron varias iniciativas para reforzar la cooperación con las autoridades locales para «facilitar la investigación, el arresto y la aprehensión de extranjeros que hayan violado la ley». ${ }^{5}$ Sin embargo, las ambigüedades sobre el papel de las autoridades locales en la aplicación de las leyes migratorias quedaron irresueltas. Para enfrentar esta situación, el Congreso introdujo dos propuestas de ley en 2003: Clear Law Enforcement for Criminal Alien Removal Act of 2003 (CLEAR Act; H.R. 2671) y Homeland Security Enhancement Act of 2003 (S. 1906), las cuales extenderían la autoridad de las policías locales y estatales para aplicar la parte civil de las leyes migratorias; a la fecha, no se han aprobado. ${ }^{6}$

El debate legislativo sobre la puesta en práctica de la ley migratoria por parte de autoridades locales y estatales ha desatado una discusión sobre cuál debe ser su papel en la tarea de garantizar el cumplimiento de las leyes migratorias. Por un lado, existen preocupaciones sobre la escasez de recursos en el ámbito local; dado que la ejecución de la ley migratoria es responsabilidad del gobierno federal, se argumenta que los recursos estatales no deben utilizarse para este propósito, además de que afectarían la labor de las policías locales en otras áreas. ${ }^{7}$ Otro pro-

${ }^{5}$ Lisa M. Seghetti, Stephen R. Viña y Karma Ester, «Enforcing Immigration Law: The Role of State and Local Law Enforcement», Congressional Research Service, The Library of Congress, 11 de marzo de 2004, p. 6 (http://www.policyalmanac.org/social_welfare/archive/immigration_ enforcement.pdf, última consulta: 21 de noviembre de 2006)

${ }^{6}$ En 2005 se reintrodujo el Homeland Security Enhancement Act como S. 1362; a la fecha tampoco ha sido aprobado.

7 Seghetti, Viña y Ester, art. cit., pp. 24-25, 27; National Immigration Forum, art. cit. 
blema que ya se ha presentado es que no existe la infraestructura suficiente en centros de detención administrados por el gobierno federal, con lo cual esta responsabilidad recaería en manos de los gobiernos locales en caso de que se involucraran ampliamente en la detención de inmigrantes indocumentados. No obstante, hay quienes argumentan que la participación de las autoridades locales contribuirá de manera importante a reducir las amenazas terroristas y aplicar la ley migratoria de forma consistente en todo el país. ${ }^{8}$ Quienes se oponen a extender las funciones de las policías locales en este ámbito argumentan que la falta de entrenamiento y experiencia por parte de las autoridades y policías puede tener como consecuencia casos de abuso de poder, violaciones a los derechos civiles de los inmigrantes y discriminación basada en perfiles raciales. ${ }^{9}$ Además, esto tendría un impacto negativo en las comunidades en aspectos tales como la cooperación para denunciar crímenes o dar testimonios, orillando a los inmigrantes a «vivir en las sombras».

Este debate ha dejado claro que hay una división entre las localidades que consideran positivo adoptar este papel y aprueban ordenanzas, órdenes ejecutivas, resoluciones y órdenes especiales para extender sus funciones y las que prefieren continuar siendo un «santuario» para los trabajadores indocumentados y los protegen por medio de prácticas como el no denunciarlos a las autoridades federales ni averiguar su estatus migratorio, excepto en investigaciones criminales. ${ }^{10}$ Un problema potencial es que estas diferencias en el ámbito local resulten en una aplicación inconsistente de la ley dependiendo de cada jurisdicción, lo cual puede generar demandas por trato desigual y discriminación. Sin embargo, hay quien argumenta que cada jurisdicción tiene diferentes necesidades y problemas, lo cual debería reconocerse en la aplicación de la ley. ${ }^{11}$

No obstante los cambios en la legislación migratoria desde 1996, el Congreso federal mantiene la autoridad exclusiva para determinar quién puede entrar y/o permanecer en territorio estadounidense y qué derechos tiene. Sin embargo, el hecho de que las autoridades locales puedan participar en las acciones para garantizar el cumplimiento de la ley migratoria bajo los criterios establecidos en la ley federal, no sólo en los aspectos criminales como se consideraba tradicionalmente, sino también en los aspectos civiles, ha suscitado un debate importante a nivel nacional sobre los alcances y límites de sus atribuciones en este ámbito. ${ }^{12}$ Las distintas interpretaciones sobre la responsabilidad y la capacidad que tienen los gobier-

\footnotetext{
${ }^{8}$ Seghetti, Viña y Ester, art. cit, p. 28.

9 Ibid., pp. 26-27.

${ }^{10}$ En 2004, el Congressional Research Service reportó que las siguientes 32 ciudades y condados tenían políticas de «santuario»: Anchorage, AK; Fairbanks, AK; Chandler, AZ; Fresno, CA; Los Angeles, CA; San Diego, CA; San Francisco, CA; Sonoma County, CA; Evanston, IL; Cicero, IL; Cambridge, MA; Orleans, MA; Portland, ME; Baltimore, MD; Takoma Park, MD; Ann Arbor, MI; Detroit, MI; Minneapolis, MN; Durham, NC; Albuquerque, NM; Aztec, NM; Rio Arriba, County, NM; Sante Fe, NM; Nueva York, NY; Ashland, OR; Gaston, OR; Marion County, OR; Austin, TX; Houston, TX; Katy, TX; Seattle, WA; y Madison, WI (Seghetti, Viña y Ester, op.cit., pp. 2-4, 24 y nota 75). A la fecha, se calcula que hay cerca de 100 "ciudades santuario» (declaradas formal o informalmente).

${ }^{11}$ Seghetti, Viña y Ester, art. cit, pp. 28-29.

12 Ibid., pp. 8-9.
} 
nos locales y estatales en este tema revelan las divisiones internas en Estados Unidos sobre cómo enfrentar las consecuencias de la migración y la frustración que existe ante una legislación migratoria disfuncional.

\section{REACCIONES EN EL ÁMBITO LOCAL}

\section{Ordenanzas e iniciativas de ley}

A partir del once de septiembre de 2001, uno de los principales temas en la discusión pública en Estados Unidos ha sido la necesidad de reformar el sistema migratorio para actualizarlo con respecto a la realidad de la migración en el país, asegurar el control de las fronteras y resolver la situación de los cerca de 11 millones de inmigrantes indocumentados que residen en su territorio. No obstante la variedad de propuestas que se han presentado, tanto por parte del Ejecutivo como de distintos congresistas y senadores, así como las manifestaciones públicas de varios sectores a favor y en contra de una reforma migratoria integral, el debate se ha caracterizado por el estancamiento y, recientemente, por la aprobación de medidas para reforzar el control de la frontera que muchos consideran insuficientes para resolver el problema de fondo.

Ante la frustración por el fracaso de las iniciativas en el Congreso y la ineficiencia de las políticas existentes, asambleas locales y gobiernos de ciudades a lo largo del país, desde San Bernardino, California hasta Suffolk County, Nueva York, han decidido tomar en sus propias manos la aprobación de leyes y ordenanzas para controlar las situaciones que enfrentan diariamente como resultado del aumento de la migración, en particular, la migración indocumentada. Cerca de 80 leyes relacionadas con la migración se aprobaron en al menos 32 estados en 2006, mientras que en el primer semestre de 2007 se presentaron cerca de 1,170 iniciativas locales a lo largo de los 50 estados de la Unión Americana. ${ }^{13}$ La mayoría se caracterizan por promover medidas restrictivas que responden a los grupos anti-inmigrantes molestos por el notable incremento de la población de hispanos en comunidades suburbanas, en las que normalmente no existían estas minorías, así como la amenaza que perciben en términos de lenguaje, cultura y valores. Por ejemplo, el alcalde de Hazleton, PA, Lou Barletta, argumenta que la migración indocumentada es la causa del detrimento en la calidad de vida en la ciudad, el aumento del crimen y las sobrecargas en escuelas y hospitales. ${ }^{14}$

13 «US State and Local Governments Respond to Federal Inaction on Immigration», Migration Information Source, Migration Policy Institute (MPI), 1 de diciembre de 2006; Summer Harlow, «Small towns play big role on immigration: Fear of persecution forces many to move», The News Journal (Wilmington, DE), 15 de octubre de 2006; «Frustrated by federal inaction, state and local governments are passing laws at a record pace», The Christian Science Monitor, 15 de junio de 2007; "Illegal Immigrants Targeted By States», The Washington Post, 25 de junio de 2007).

14 «One Mayor's Controversial Plan to Deal with Illegal Immigration», 60 Minutes, CBS News, 19 de noviembre de 2006. 
Para desincentivar la llegada de más inmigrantes indocumentados, o forzar la salida de quienes ya se encuentran en estas comunidades, algunos gobiernos locales han aprobado medidas para evitar que se alquilen viviendas a personas que no tengan estatus legal y se sancione a quien lo haga (tal es el caso de las iniciativas introducidas en Hazleton y Altoona, PA; Escondido y San Bernardino, CA; Cobb County y Cherokee County, GA; Manassas y Culpeper, VA, Riverside, NJ; Farmers Branch, TX; Harrington y Elsmere, DE; Avon Park, FL; y Valley Park, MO; entre otras) e incluso han desalojado a residentes que habitan en viviendas sobrepobladas -a quienes se sospecha de ser inmigrantes indocumentados (por ejemplo, en Farmingville, NY). Otras iniciativas proponen que se refuercen los controles y se aumenten las sanciones a empleadores para evitar que contraten a trabajadores indocumentados. Algunos ejemplos se han presentado en las siguientes ciudades y condados: Suffolk County, NY; Altoona y Hazleton, PA; Riverside, NJ; Beaufort County y Dorchester County, SC; Harrington y Elsmere, DE; Herndon y Culpeper, VA; Palm Bay, FL; Valley Park, MO; Farmers Branch, TX; y a nivel estatal: Colorado, Arizona, Alabama, Arkansas, Georgia, Missouri y Pennsylvania. ${ }^{15}$

Varios estados también han propuesto que se limite el acceso a servicios públicos a quienes no sean ciudadanos o residentes legales (por ejemplo, en el caso de Colorado ${ }^{16}$ y Arizona ${ }^{17}$ ) y que se impida a los inmigrantes indocumentados obtener licencias para operar negocios (por ejemplo, en Herndon, VA y Hilton Head, SC). Asimismo, como se detallará más adelante, varias localidades han tomado medidas para limitar la presencia de trabajadores jornaleros en la vía pública y evitar el establecimiento de centros de contratación. Finalmente, algunos estados y ciudades

15 Véase «Sistema de Monitoreo sobre Iniciativas Locales en Materia de Control Migratorio», Instituto de los Mexicanos en el Exterior, México D.F., julio 2007 (http://www.ime.gob.mx/ smilcm.htm)

${ }^{16}$ Como parte de la elección intermedia del 7 de noviembre de 2006, en el estado de Colorado, el electorado votó sobre varias iniciativas para controlar la migración indocumentada. Las propuestas fueron aprobadas por un margen pequeño. El referéndum $\mathrm{H}$, el cual niega el derecho a dar créditos de impuestos a empleadores que contraten a trabajadores indocumentados, fue aprobado con 50.8\%. El referéndum K, el cual obliga al Procurador General demandar al gobierno federal por falta de cumplimiento de las leyes migratorias, fue aprobado por $56 \%$. Los legisladores que auspiciaron estas propuestas opinaron que la falta de apoyo quizás se debió a que la asamblea local aprobó 17 medidas para controlar la migración indocumentada durante su periodo de sesiones. Otros opinan que tiene que ver con que los electores no entienden bien este tipo de propuestas o no consideran que el tema migratorio es una prioridad (Fernando Quintero, «Illegal immigration measures pass underwhelmingly», Rocky Mountain News, 9 de noviembre de 2006).

${ }^{17}$ Cuatro propuestas migratorias que se sometieron a votación en el estado de Arizona el 7 de noviembre fueron aprobadas. La Propuesta 103, una de las más controvertidas, declara al inglés como idioma oficial del estado (aunque habría excepciones en casos como procedimientos legales). La Propuesta 300 elimina la posibilidad de que inmigrantes indocumentados tengan acceso a fondos estatales para educación para adultos y cuidado de niños. La Propuesta 100 impide que los inmigrantes indocumentados puedan salir de la cárcel bajo fianza para evitar que salgan del país antes de un juicio. La Propuesta 102 limita las posibilidades de que los inmigrantes indocumentados presenten una demanda por daños y perjuicios, limitando su recompensa sólo a daños específicos, lo cual protegería a grupos como empleadores de pagar altas penas por casos de empleados accidentados en el trabajo. 
han planteado declarar al inglés como idioma oficial para la emisión de documentos del gobierno, como son los casos de Arizona; Cherokee County, GA; Taneytown, MD; Valley Park, MO; Pahrump, NE; Hazleton, PA; Nashville, TN; Farmers Branch y Friendswood, TX; Culpeper y Herndon, VA. Aunque se ha argumentado que las medidas sobre el idioma no tienen un efecto directo sobre la comunidad inmigrante, ni modifican significativamente las prácticas establecidas, se consideran un acto simbólico para dar una señal a estos grupos de que no son bienvenidos. Dadas estas implicaciones, en casos como el de Nashville, el alcalde decidió vetar una ordenanza de «sólo inglés» por considerarla mal-intencionada, inconstitucional e innecesaria ${ }^{18}$ pero en otros estados y ciudades (por ejemplo, Arizona; Beaufort County, SC; Cherokee County, GA; Hampshire, IL; Taneytown, MD; y Bridgeport, PA) estas medidas han sido aprobadas, en muchos casos por unanimidad.

Para algunos críticos, estas leyes y ordenanzas no sustituyen las acciones que se requieren para una reforma seria e integral $y$ «no logran imponer orden ni consistencia en donde realmente se requiere: a nivel federal»; ${ }^{19}$ al contrario, dejan claro que existe un vacío legal -o al menos en el cumplimiento de la ley-, crean comunidades de inmigrantes temerosos y provocan tensiones sociales, dificultan las actividades de empresas y negocios que dependen de su mano de obra y crean un mercado de vivienda hostil, imponiendo responsabilidades migratorias a los caseros, e incluso, en algunos casos, duplican leyes existentes. De acuerdo con Witold J. Walczak, director de la American Civil Liberties Union (ACLU) de Pennsylvania, «la reforma migratoria es un asunto importante, pero si cada pequeña ciudad como Hazleton a lo largo de los 50 estados decide crear sus propias reglas sobre migración, el resultado va a ser un problema aún mayor». ${ }^{20}$ Quienes proponen o apoyan estas medidas argumentan que los estados y ciudades simplemente están fortaleciendo el cumplimiento de las leyes y ordenanzas que ya existen -no tratando de reemplazar las leyes federales-, pues no pueden seguir esperando indefinidamente a que el gobierno federal tome acciones al respecto. ${ }^{21}$

Esta situación ha dado lugar a un debate en las cortes locales y estatales que algunos consideran podría llegar hasta la Suprema Corte de Justicia. Varios expertos legales argumentan que estas leyes son inconstitucionales pues la ley federal prohíbe que los estados y ciudades impongan sanciones criminales o civiles por migración indocumentada que sean mayores a las que adopte el Congreso. ${ }^{22} \mathrm{La}$ American Civil Liberties Union (ACLU) y el Puerto Rican Legal Defense and Education

\footnotetext{
18 "A smart veto from the mayor of Nashville», Houston Chronicle, 13 de febrero de 2007.

19 "Hazy Days of Immigration», Editorial, The New York Times, 20 de julio de 2006 (véase también «Bad plan, Escondido», Editorial, Los Angeles Times, 2 de noviembre de $2006 \mathrm{y}$ «Immigration in Herndon", Editorial, Washington Post, 2 de octubre de 2006; A18).

${ }^{20}$ Citado por Michael Rubinkam, «Pa. Town «s Immigration Law Challenged», The Associated Press, 30 de octubre de 2006.

${ }^{21}$ Véase Harlow, art. cit.

${ }^{22}$ Erik Schlezig, "Expert: State Immigrant Laws Might Fail», The Associated Press, 19 de agosto de 2006.
} 
Fund (PRLDEF), junto con otras organizaciones, han iniciado demandas en contra de varias de estas iniciativas. En algunos casos han logrado bloquear su aplicación $y$, en otros, las ordenanzas han sido revocadas.

Las demandas, en las que se representa ya sea a grupos de inmigrantes o a residentes legales, caseros y dueños de negocios que, o han sido discriminados o enfrentan pérdidas económicas como resultado de las ordenanzas, se basan en argumentos como el hecho de que el gobierno federal tiene el poder exclusivo de regular la migración «y aunque las ciudades estén en desacuerdo con las políticas federales o la forma en que el gobierno federal está desempeñando su trabajo respecto a la migración [...las] miles de ciudades y municipios en el país no pueden asumir ese papel..$^{23} \mathrm{~A}$ su vez, argumentan que las ordenanzas violan el derecho a vivienda y los derechos civiles que prohíben la discriminación basada en el perfil racial o étnico; que son discriminatorias específicamente en contra de los hispanos y son inaplicables porque implican, por ejemplo, que toda persona que sea o parezca extranjero es sospechosa de encontrarse en el país sin autorización, aunque su estatus migratorio sea regular. ${ }^{24}$

Además de las demandas en contra de las ordenanzas, esta situación ha provocado protestas y movilizaciones por parte de distintos sectores -iglesias, sindicatos, grupos de negocios, organizaciones defensoras de inmigrantes, organizaciones latinas como LULAC y MALDEF y grupos de ciudadanos. En el caso de Farmers Branch, por ejemplo, en noviembre de 2006, voluntarios del grupo «Deja a los votantes decidir», realizaron un recorrido por la ciudad, con el objetivo de reunir las 700 firmas necesarias para que la ordenanza 2892, que multaría a caseros que no verificaran el estatus legal de sus inquilinos, fuera reconsiderada y sujeta a una nueva votación. $^{25}$

Varias localidades han dejado pendientes las ordenanzas en espera de los resultados de los juicios en otros lugares o las han rescindido ante las presiones de ACLU y otros grupos (por ejemplo en Manassas, VA; San Bernardino, CA; Palm Bay y Avon Park, FL y Elsmere, DE). En el caso de Hazleton, PA, el cual ha sido ampliamente divulgado en los medios, un juez federal bloqueó la ordenanza «Illegal Immigration Relief Act», que impondría sanciones a propietarios que alquilen viviendas a inmigrantes indocumentados y a empresas que los contraten. La demanda, presentada por el PRLDEF, la ACLU y otros grupos, argumenta que Hazleton ha sobrepasado su autoridad con respecto al control de la migración y que la ordenan-

${ }^{23}$ Harlow, art. cit.

${ }^{24}$ Por ejemplo, en el caso de la ciudad Escondido, California ante la demanda de la ACLu, un juez federal bloqueó la aplicación de la ordenanza para imponer sanciones a caseros que alquilen cuartos o viviendas a inmigrantes indocumentados, argumentando que la ley está sujeta escrutinio legal ya que puede infligir daños irreparables tanto en los inquilinos como en los caseros (Elliot Spagat, "Anti-Illegal Immigrant Law Put on Hold», The Associated Press, 16 de noviembre de 2006; David Fried, "Escondido has until end of December to respond to lawsuit», North County Times, 27 de noviembre de 2006).

${ }^{25}$ Luis Ángel Galván, «Consideran positivo el avance en la recolección de firmas», Diario La Estrella, 29 de noviembre de 2006. 
za es discriminatoria. Un juez de distrito dictó una orden temporal para restringir la aplicación de la ordenanza, que habría entrado en vigor a partir del primero de noviembre. Sin embargo, el alcalde de la ciudad ha declarado que no se dará por vencido ante las cortes locales y, de ser necesario, llevará el caso hasta la Suprema Corte.

A pesar de que muchas de las ordenanzas no han entrado en vigor o han sido revocadas, han cumplido uno de sus objetivos principales, pues muchos inmigrantes hispanos han abandonado estas localidades ante la discriminación que enfrentan, lo cual se ha visto directamente reflejado en pérdidas económicas para negocios de la zona. Según el Alcalde Barletta, aproximadamente 5,000 hispanos han salido de Hazleton desde que se comenzó a discutir la ordenanza. Los dueños de negocios señalan que sus ventas han disminuido entre $20 \%$ y $50 \%$ y al menos dos establecimientos tuvieron que cerrar. En el caso de Riverside, Nueva Jersey, cuya principal población de inmigrantes indocumentados es de origen brasileño, se estima que las pérdidas económicas por la salida de inmigrantes llegan hasta $40 \% .{ }^{26}$ Se ha argumentado que estos costos serán resentidos por las comunidades pues verán la pérdida de los beneficios económicos que aportan los inmigrantes. ${ }^{27}$ Sin embargo, algunos habitantes de Hazleton lo consideran un cambio positivo y argumentan que, gracias a la ordenanza, «los narcotraficantes, asesinos y ladrones» han comenzado a salir de la ciudad. ${ }^{28}$

\section{Respuestas a la presencia de trabajadores jornaleros ${ }^{29}$}

En los últimos años ha sido notoria la creciente presencia de trabajadores jornaleros ("day-laborers») en suburbios estadounidenses en donde tradicionalmente no había un alto porcentaje de inmigrantes latinos. Esta tendencia refleja los cambios generales en los patrones migratorios, los cuales se caracterizan por la expansión de redes de inmigrantes hacia ciudades pequeñas, sobre todo en el centro y el este de Estados Unidos, en donde actualmente hay más oportunidades de empleo informal en los sectores de construcción y trabajo doméstico.

El crecimiento de la población inmigrante en estas comunidades suburbanas y su acceso limitado a una estructura formal de trabajo ha provocado reacciones

\footnotetext{
${ }^{26}$ Harlow, art. cit.

${ }^{27}$ En el caso de Hazleton, el aumento de la población hispana había sido resultado de un programa instrumentado por el estado de Pennsylvania hace cerca de una década para atraer nuevos negocios al ofrecer incentivos fiscales. Con ello aumentó el número de fábricas, centros de distribución y oficinas, y se crearon más de 5,000 empleos, en su mayoría para trabajadores de baja calificación, lo cual impulsó el crecimiento de la población de hispanos de $5 \%$ de la población total de la ciudad a 30\%. Véase Ellen Barry, "City's immigration law turns back clock», Los Angeles Times, 9 de noviembre de 2006 y «One Mayor's Controversial Plan To Deal With Illegal Immigration», art. cit.

${ }^{28}$ Barry, "City's immigration law turns back clock», art. cit.

${ }_{29}$ Parte de esta sección se publicó bajo el título "Jornaleros (Day-Laborers)», Boletín Temático, Instituto de los Mexicanos en el Exterior, vol. 2, núm. 1, abril de 2006 (http://www.ime.gob.mx/ noticias/boletines_tematicos/Jornaleros.pdf)
} 
por parte de la población local y de sus representantes políticos. El debate se enfoca principalmente al problema de que los jornaleros se reúnan en las calles o cerca de tiendas de autoservicio ${ }^{30}$ en espera de ser contratados, y que en algunos casos ocupen viviendas sobrepobladas en condiciones insalubres.

Las respuestas de cada localidad han variado dependiendo de las características del problema y los grupos involucrados. En algunas ciudades se ha apoyado la creación de centros de contratación para jornaleros con financiamiento del gobierno local o de organizaciones comunitarias como una solución temporal (tal es el caso de Houston, TX; ${ }^{31}$ Denton, TX;32 Fort Worth, TX; Chandler, AZ;33 Phoenix, AZ; Laguna Beach, CA; Burbank, CA; ${ }^{34}$ Jupiter, FL; ${ }^{35}$ Herndon, VA; Lakewood, NJ) y otras medidas para auxiliar a los jornaleros (como en el caso de las líneas telefónicas para reportar abusos en Santa Cruz, $\mathrm{CA}^{36}$ ), además de respaldar la labor de los centros de apoyo para trabajadores (worker centers). ${ }^{37}$ En otras, se ha dado una fuerte ola anti-inmigrante que incluye manifestaciones en contra de los jornaleros, protestas por parte del grupo «Minuteman Project», desalojo de viviendas

${ }^{30}$ De acuerdo al estudio de Valenzuela et al. (2006) entre 25 y 200 trabajadores jornaleros acuden diariamente a centros de reunión en busca de trabajo. $79 \%$ de estos centros de contratación son informales: $24 \%$ de los jornaleros se reúnen frente a negocios, $22 \%$ frente a tiendas de productos para trabajo en el hogar, $10 \%$ en expendios de gasolina y $8 \%$ en calles con alto tránsito. Sólo uno de cada cinco jornaleros (21\%) busca trabajo en centros de contratación formales para jornaleros.

${ }^{31}$ En la ciudad de Houston, Texas operan varios centros de contratación para jornaleros, financiados por el gobierno de la ciudad. Sin embargo, algunos trabajadores continúan solicitando trabajo en las calles, lo cual ha llevado a arrestos y críticas por parte de la población local.

32 En la ciudad de Denton, Texas ha habido protestas por parte de miembros del grupo Minuteman Project, el cual ha organizado una campaña nacional para poner en evidencia a los empleadores que contraten a jornaleros e impedir el establecimiento de más centros de contratación. En el caso de Denton, esto no ha impedido el continuo flujo de trabajadores y contratistas en el centro de contratación de la localidad.

${ }^{33}$ En la ciudad de Chandler, Arizona un grupo de la iglesia metodista opera el centro de contratación para trabajadores «Light and Life Day Labor Center» para evitar las quejas de tráfico que provocaba la presencia de jornaleros en calles con altos niveles de tránsito. Como parte de las acciones para enfrentar este problema e incentivar el uso del centro de contratación, la policía local comenzó a multar a los empleadores que se detuvieran en la avenida Arizona para contratar a jornaleros. El centro de Chandler ha promovido campañas de educación para informar a los jornaleros sobre sus derechos y ha buscado la colaboración con el consulado de México para prevenir abusos laborales a los jornaleros.

${ }^{34}$ En la ciudad de Burbank, California, las autoridades locales solicitaron a la tienda Home Depot habilitar un centro de contratación para jornaleros con el fin de eliminar la presencia de grupos de trabajadores en las banquetas y en el estacionamiento del establecimiento, así como proporcionarles un espacio adecuado para negociar con los empleadores. Un grupo católico se encarga de operar el centro, el cual fue el primero de este tipo en todo el país. Recientemente, otras ciudades (Woodland Hills, Monrovia, Glendale en California y Washington, D.C.) han requerido que el Home Depot establecer centros similares y/o pagar una cuota para financiar su operación.

${ }^{35}$ En la ciudad de Jupiter, Florida se aprobó recientemente la creación de un centro de contratación para jornaleros «Jupiter Neighborhood Resource Center», el cual ha sido apoyado por sindicatos de la región.

${ }^{36}$ En la ciudad de Santa Cruz, California, la policía local estableció una línea telefónica directa para que los jornaleros que trabajan en la zona reporten abusos por parte de empleadores. El proyecto garantiza que la policía no hará averiguaciones sobre el estatus migratorio de los trabajadores.

${ }^{37}$ Véase Fine, 2005. 
sobrepobladas, detenciones de jornaleros, ordenanzas para prohibir que los jornaleros ofrezcan sus servicios en la vía pública (Glendale, CA; Gaithersburg, MD), iniciativas de ley anti-vagabundeo (anti-loitering), acoso a los empleadores que contratan esta mano de obra y oposición al establecimiento de centros de contratación. ${ }^{38}$

El debate sobre los centros de contratación para jornaleros se centra en la discusión sobre si estos establecimientos deberían ser financiados con recursos públicos. Quienes apoyan el uso de fondos del estado o la ciudad para el establecimiento y la operación de los centros argumentan que es necesario que el gobierno local reconozca la realidad de demanda y oferta de trabajo y provea los medios para resolver el problema, así sea de manera temporal. Quienes se oponen a estos centros argumentan que el uso del presupuesto del estado o la ciudad para su operación, significa apoyar la migración indocumentada e implica utilizar el dinero de los ciudadanos que pagan impuestos, lo cual representa una violación a la ley federal. ${ }^{39}$ Algunos grupos proponen que la tienda de productos para construcción y remodelación, Home Depot, la cual ha sido el centro de reunión para jornaleros en varias localidades, debería hacerse responsable de subsidiar estos centros.

En las ciudades de Farmingville, NY y Herndon, VA, se han presentado algunos de los casos más notorios respecto al debate sobre los jornaleros. Las características de la discusión en estas localidades son representativas de las situaciones más polémicas que se han dado a lo largo del país, aunque cabe tomar en cuenta que en muchas ciudades, incluso dentro de estos dos estados, hay una actitud de tolerancia hacia los jornaleros y poca oposición a la creación de centros de contratación.

\section{Farminguille, NY}

El poblado de Farmingville, dentro de la ciudad de Brookhaven, se encuentra en el condado de Suffolk del área suburbana de Long Island, Nueva York. En 1970 esta zona era 95\% anglosajona; actualmente, está poblada por más de 300,000 inmigrantes latinos (la mayoría son salvadoreños, seguidos por mexicanos, hondureños, colombianos y ecuatorianos) (Gordon, 2005). La tensión social que ha

\footnotetext{
${ }^{38}$ Por ejemplo, en la ciudad de Tucson, Arizona recientemente la policía local ha comenzado a endurecer sus medidas de control en contra de los jornaleros en las calles respondiendo a quejas de algunos vecinos. A su vez, en los últimos meses, la policía de Cicero (un suburbio de Chicago, Illinois) ha arrestado a más de 50 jornaleros en la zona, lo cual ha provocado reacciones por parte de grupos de defensa de jornaleros. Por su parte, la tienda Home Depot del suburbio de Cicero ha sido acusada de apoyar la migración indocumentada, por lo que contrató a un mediador para resolver la disputa sobre jornaleros que se reúnen a buscar trabajo cerca del establecimiento.

${ }^{39}$ Véase, por ejemplo, Jennifer Delson, «Laguna Beach Is Sued Over Day Laborer Center», Los Angeles Times, 4 de octubre de 2006.
} 
resultado del crecimiento de la población de inmigrantes en una comunidad tradicionalmente conservadora, como es el caso de Farmingville, es percibida por varios grupos como una amenaza a sus valores comunitarios. Como resultado de las percepciones negativas sobre la reunión de jornaleros en las calles en espera de sus contratistas y la evidencia de viviendas sobrepobladas, se han creado coaliciones anti-inmigrantes $y$, en dos casos extremos, han ocurrido ataques violentos en contra de los jornaleros y sus familias. ${ }^{40}$ La situación de Farmingville ha recibido un alto grado de atención por parte de los medios de comunicación e incluso fue el tema de un documental titulado Farmingville, ganador del Premio Especial del Jurado 2004 del festival de cine de Sundance. ${ }^{41}$

Una de las propuestas para resolver el problema fue la de crear centros de contratación financiados en parte por el gobierno local, como es el caso de los centros de contratación de Freeport, Huntington Station y Long Island. En el caso de Farmingville, el ejecutivo del condado de Nassau, Thomas Suozzi (D), apoyó la creación de centros de contratación como una forma de resolver el problema, siguiendo el ejemplo de la ciudad de Glen Cove, que en 1994 se convirtió en la primera ciudad de la costa este en abrir un centro de contratación para jornaleros. Sin embargo, la propuesta para crear el centro en Farmingville fue rechazada por el Concejo de la Ciudad ante la presión y las protestas de varios grupos organizados de la comunidad.

Por su parte, el ejecutivo del condado, Steve Levy (D), respaldó una serie de políticas restrictivas en contra de los jornaleros. Esto incluyó el desalojo selectivo de viviendas sobrepobladas sin aviso previo, ni el otorgamiento del periodo de gracia que establece la ley para rectificar la situación o reubicar a los inquilinos en otra vivienda. ${ }^{42}$ Frente al desalojo de cerca de 11 viviendas y 200 personas -en su mayoría inmigrantes latinos-, durante el verano de 2005, los centros para jornaleros y grupos activistas de la zona, entre ellos el Workplace Project, ofrecieron tiendas de campaña para los jornaleros desalojados y a manera de protesta denominaron la zona de acampado como Levyville. Por su parte, el PRLDEF presentó una demanda ante la corte local argumentando que la política de desalojo no se hizo conforme a derecho. El prLDef llevó el caso a una corte federal de Long Island, la cual declaró el 16 de diciembre de 2005, que las autoridades de la ciudad de Brookhaven discriminaron en contra de los jornaleros latinos al instrumentar su política de desalojo y presentó una orden en la que exige que se dé una notificación previa a los inquilinos de la vivienda sujeta a desalojo.43

${ }^{40}$ En 2000, dos hombres llevaron a un par de trabajadores jornaleros a una bodega prometiéndoles trabajo. Al llegar al lugar, los golpearon con herramientas, resultando en graves heridas. Ambos atacantes fueron declarados culpables de ataques motivados por racismo. En 2003, un grupo de adolescentes atacaron la vivienda de una familia mexicana con fuegos artificiales; no se reportaron heridos pero la casa resultó seriamente dañada.

${ }^{41}$ El documental fue dirigido y producido por Catherine Tambini y Carlos Sandoval.

${ }^{42}$ Otros casos de desalojo de viviendas sobrepobladas, generalmente habitadas por inmigrantes indocumentados, se han presentado en Virginia, Massachussets y Georgia.

${ }^{43}$ Véase «PRLDEF Continues Fight For Day Laborers», en http://www.prldef.org/Civil/mayor\%20letter.pdf (última consulta: marzo de 2006). 
Recientemente, el debate sobre los jornaleros se ha extendido a otras comunidades del estado de Nueva York, como es el caso de las ciudades de Brewster, Mamaroneck, Patchogue, Greenport y East Hampton, en donde se discute la posibilidad de establecer centros de contratación ante las reacciones xenófobas de algunos grupos molestos por la presencia de jornaleros en las calles. En el estado de Nueva Jersey, destaca la situación similar de ciudades como Freehold, Morristown, Lakewood y Passaic. Mientras que el PRLDEF y otros grupos han argumentado que los jornaleros tienen el derecho constitucional de buscar trabajo en zonas públicas, grupos anti-inmigrantes como United Patriots of America han organizado manifestaciones bajo el lema «Stop the Invasion»-como parte de una campaña de protestas en varios estados-, con el fin de presionar a las autoridades locales para que instrumenten políticas migratorias restrictivas en contra de los jornaleros.

Otro caso destacado en el área es el de Danbury, Connecticut en donde el alcalde de la ciudad, Mark Boughton (R), inició una campaña para reforzar la política de control a los jornaleros, respondiendo a las demandas de grupos anti-inmigrantes como Connecticut Citizens for Immigration Control. A su vez, el alcalde Boughton se unió al ejecutivo del condado de Suffolk, Steve Levy (D), para fundar el grupo "Alcaldes y Ejecutivos por la Reforma Migratoria» (Mayors and Executives for Immigration Reform) cuyo objetivo es presionar al gobierno federal para que compense con mayores recursos a las localidades que enfrenten costos por el crecimiento de la población indocumentada. La organización, que incluye a más de 60 funcionarios electos de 30 comunidades en Estados Unidos, refleja la intensidad del debate en el ámbito de los gobiernos locales y su frustración respecto a la ejecución de las leyes migratorias a nivel federal, ante lo cual plantean soluciones alternativas propias. $^{44}$

\section{Herndon, Virginia}

En la década de 1990, el número de latinos en la ciudad de Herndon, Virginia, creció $264 \%$, lo cual significa que actualmente cuatro de cada diez residentes de la localidad son extranjeros. Los inmigrantes -documentados e indocumentados- han llegado a esta zona para ocupar trabajos de construcción y mejora del hogar o laborar como empleados domésticos. Hasta diciembre de 2005, entre 60 y 100 inmigrantes latinos se reunían cada mañana cerca de una tienda de autoservicio 7-Eleven en espera de contratistas, en un ambiente de desorganización y condiciones insalubres, lo cual provocó reacciones por parte de la población local.

La propuesta del alcalde de la ciudad, Michael O'Reilly, de establecer un centro de contratación de jornaleros tuvo como consecuencia reclamos y críticas de grupos anti-inmigrantes e incluso del candidato republicano a gobernador de

\footnotetext{
${ }^{44}$ Véase «Long Island, The Wrong Path on Immigration», Editorial, The New York Times, 11 de marzo
} de 2006. 
Virginia, Jerry W. Kilgore, lo cual influyó en que se detuviera la iniciativa. No obstante, un grupo de iglesias y líderes comunitarios del Project Hope and Harmony, apoyado por la organización HEART-Herndon Embraces All With Respect and Tolerance, logró que el Concejo de la Ciudad aprobara una propuesta para financiar un centro de contratación que consistiría en una unidad móvil en un local desalojado. La aprobación de esta propuesta generó una ola de críticas y protestas en la ciudad, además de una demanda legal por parte de un grupo opositor, pero no impidió que el centro comenzara a funcionar en diciembre de 2005. Al poco tiempo se redujeron las protestas, aunque coaliciones como Help Save Herndon continúan promoviendo acciones con el fin clausurar el centro. Hasta ahora, éste ha operado de manera eficiente por medio de boletos de rifa para asignar a los trabajadores de manera ordenada y tiene un estricto código de conducta para evitar acusaciones en su contra. 45

La experiencia de Herndon ha influido en otros casos similares en Virginia y los estados vecinos de Maryland y Washington D.C., principalmente en las ciudades de Gaithersburg, Arlington, Silver Spring y Wheaton, en donde, pese a la oposición de ciertos grupos, operan centros de contratación con resultados positivos en términos de evitar la reunión desordenada de grupos de jornaleros en las calles y afuera de tiendas. En el caso de Silver Spring, Maryland, mientras que hace 10 años hubo protestas del grado de las de Herndon, poco a poco la población local se ha vuelto más tolerante hacia el $40 \%$ de sus residentes inmigrantes y han apoyado el establecimiento de centros para jornaleros. En el condado de Prince George, Maryland, también se está considerando habilitar un centro similar. El consenso general es que los centros de contratación son preferibles a la situación alternativa que implica desorganización y tensión social como resultado de la concentración de jornaleros en estacionamientos, iglesias o esquinas transitadas.

\section{Demandas exitosas}

Recientemente, el PRLDEF y la ACLU han ganado importantes juicios en las demandas que han presentado junto con otros grupos en casos de discriminación en contra de los jornaleros y violación de sus derechos civiles. Estas victorias legales sientan importantes precedentes en materia de jurisprudencia con respecto a la

\footnotetext{
${ }^{45}$ A partir de la elección del nuevo Concejo de la ciudad (con sólo 2 de sus miembros reelectos), los nuevos miembros, en su mayoría opositores del centro, han propuesto reemplazar al grupo religioso (Reston Interfaith) que opera el Herndon Official Workers Center. En su lugar, sugieren que el centro debe ser operado por una compañía privada que exigirá a los jornaleros comprobar que se encuentran en el país legalmente y tienen permiso de trabajo. Actualmente, el centro sólo exige que los trabajadores den su nombre y proporcionen su dirección y teléfono. La organización Casa of Maryland, la cual opera otros tres centros para jornaleros, tampoco pide ningún documento oficial a los trabajadores. A su vez, las autoridades de Herndon están considerando la posibilidad de reubicar el centro, el cual se encuentra próximo a la zona residencial de Loudoun County (véase Bill Turque, «New Group Is Sought to Run Labor Center», The Washington Post, 31 de octubre de 2006).
} 
protección de los derechos humanos y laborales de los trabajadores jornaleros y de los inmigrantes indocumentados en general.

En el caso de Freehold, Nueva Jersey, en noviembre de 2006, el PRLDEF logró establecer un acuerdo con el gobierno de la ciudad tras un juicio de tres años con respecto a la ordenanza legal emitida por las autoridades locales que prohibía a los jornaleros solicitar trabajo en lugares públicos, entre otras medidas que se consideraban discriminatorias contra esta población. El acuerdo estipula que el condado no interferirá en el uso legal de la propiedad pública, lo cual incluye permitir que los empleadores recojan a jornaleros en sitios públicos; no prohibirá el ejercicio de la libertad de expresión, lo cual incluye permitir que los jornaleros soliciten empleo; no aplicará el código de inspección de vivienda sin que el residente sea notificado con anticipación sobre sus derechos y que se cuente con el consentimiento del mismo, y no habrá participación de personal policiaco en estas actividades; reembolsará las multas impuestas a las personas involucradas y cubrirá las cuotas de sus representantes legales. ${ }^{46}$ Un fallo similar se emitió en Redondo Beach, California, en mayo de 2006 en el que un juez federal prohibió a la policía de la ciudad arrestar a trabajadores jornaleros por solicitar trabajo en la vía pública. ${ }^{47}$

En Mamaroneck, Nueva York, en septiembre de 2006, el PRLDEF (representando a cuatro jornaleros afectados) presentó una demanda denunciando el acoso por parte de autoridades y policías de la ciudad. La demanda acusaba a las autoridades de violar los derechos de libre asociación y libertad de expresión al cerrar el acceso a un centro de contratación establecido en Columbus Park y monitoreando las actividades de los jornaleros, así como las de sus empleadores. ${ }^{48}$ En noviembre de 2006, un juez federal determinó que las autoridades de la ciudad discriminaron en contra de trabajadores jornaleros de la zona al realizar actividades de patrullaje, cerrar un centro de contratación y multar a empleadores que trataban con los jornaleros. Sugirió a las partes involucradas presentar propuestas para llegar a un acuerdo, cuya fecha límite ha sido extendida varias veces a lo largo de 7 meses -la última versión, presentada el 12 de junio de 2007, es similar al acuerdo de Freehold. Ante este resultado positivo, el presidente del PRLDEF, César Perales, declaró que estas demandas envían un fuerte mensaje a los gobiernos locales a lo largo del país

${ }^{46}$ Edwin Andrés Martínez Tutek, «Compensarán a jornaleros de NJ», El Diario, NY, 15 de noviembre de 2006; «Triunfo para jornaleros de Freehold», Diario Hoy, 14 de noviembre de 2006.

${ }^{47}$ Fernanda Santos, "Day Laborers' Lawsuit Casts Spotlight on a Nationwide Conflict», The New York Times, 17 de septiembre de 2006.

${ }^{48}$ La demanda original también acusaba a las autoridades de la ciudad por acoso y discriminación en contra de los trabajadores de origen hispano. El Juez de la Corte Distrital, Colleen McMahon, declaró que el estatus migratorio de los jornaleros era irrelevante para determinar el resultado de la demanda por acoso pero que los abogados de la ciudad tenían derecho a preguntar sobre su estatus migratorio para determinar cuáles serían sus derechos en este caso de acuerdo con la Primera Enmienda. El abogado de los jornaleros, Alan Levine, del PRLDEF, se pronunció en desacuerdo con esta determinación y retiró las acusaciones referentes a la Primera Enmienda para evitar que se cuestionara a los jornaleros sobre su estatus legal (Candice Ferrette, "Mamaroneck day laborers drop First Amendment part of suit», The Journal News, 12 de septiembre de 2006). 
respecto a que los jornaleros tienen derechos y si lo ignoran, tendrán que enfrentar las consecuencias. ${ }^{49}$

\section{EL DEBATE LOCAL COMO TERMÓMETRO DEL DEBATE NACIONAL}

Los resultados de algunas elecciones locales recientes dejan clara la división que existe sobre cómo responder a la migración indocumentada. Las autoridades enfrentan una tensión constante entre la necesidad de garantizar la oferta de mano de obra para empresas y negocios locales y las presiones de grupos de ciudadanos que perciben efectos sociales negativos como resultado de la presencia de nuevos grupos de extranjeros en sus localidades. En opinión de Tamar Jacoby, las «cruzadas anti-inmigrantes» provocan que los dueños de negocios, los empleadores y los grupos moderados, se distancien de los políticos que proponen medidas restrictivas y no toman en cuenta la demanda de trabajadores que existe en diversos sectores de la economía..$^{50}$ Este argumento es sostenible en casos en los que se han presentado efectos contraproducentes -y sorpresivos- en campañas electorales basadas en una posición anti-inmigrante, como fue el caso de las elecciones locales en Long Island ${ }^{51}$ y Virginia ${ }^{52}$ en noviembre de 2005; sin embargo, en otros casos se ha castigado a políticos que apoyan explícitamente medidas a favor de los inmigrantes indocumentados, como fue el caso del Alcalde O'Reilly en Herndon, VA, quien perdió la reelección en mayo de 2006.53

${ }^{49}$ Fernanda Santos, «Village Officials Harassed Day Laborers, Judge Rules», The New York Times, 21 de noviembre de 2006.

${ }^{50}$ Tamar Jacoby, "The Immigration Temptation», The Weekly Standard, vol. 11, núm. 18, 23 de enero de 2006.

${ }^{51}$ En Long Island, los candidatos que perdieron la elección local de noviembre de 2005 generalmente fueron quienes apelaban al miedo y al resentimiento anti-inmigrante en sus campañas. Este resultado fue contrario a las expectativas de algunos analistas en el sentido de que la mayoría de la población preocupada por el problema de los jornaleros votaría por candidatos que promovieran medidas restrictivas. De acuerdo con un editorial del diario The New York Times, el hecho de que perdieran la elección llevó a la conclusión de que la mayoría de la población en esta localidad prefiere mantener una discusión sobria sobre este tema complejo y plantear soluciones amplias en lugar de enfrentar el asunto con medidas agresivas ("Where's the Outrage?», The New York Times, Editorial, 20 de noviembre de 2005).

${ }^{52}$ En Virginia, el candidato republicano, Jerry W. Kilgore, inesperadamente perdió la elección de noviembre de 2005 por el gobierno del estado. Su campaña estaba basada en argumentos conservadores y a favor de medidas restrictivas contra los inmigrantes indocumentados. Sin embargo, el resultado de las elecciones mostró que aunque la población es mayoritariamente republicana y está preocupada por el problema de los jornaleros y la migración indocumentada, prefiere "políticas moderadas y pragmáticas que propongan soluciones factibles» ("The Gop's Missed Lesson", Editorial, Washington Post, 17 de diciembre de 2005; Linda Chávez, «Fellow Republicans, Open Your Doors", The New York Times, 17 de noviembre de 2005).

${ }^{53}$ En mayo de 2006, el alcalde de Herndon, Michael O'Reilly, perdió la reelección para gobernador frente a un residente local, Steve DeBenedittis, quien se había pronunciado en contra del centro de contratación para jornaleros que se estableció en diciembre de 2005 con el apoyo de O'Reilly. A su vez, los miembros del Concejo de la Ciudad de Herndon fueron reemplazados por opositores al centro de contratación. Desde el inicio de sus labores han promovido una campaña agresiva en 
Este debate también ilustra que las posiciones sobre el tema de los jornaleros -y la migración indocumentada en general- no están determinadas por afiliación partidista pues incluso al interior de los partidos Demócrata y Republicano hay opiniones divididas sobre el tema. Un ejemplo de este situación fueron las elecciones intermedias de noviembre de 2006, en donde ciertamente el tema de la reforma migratoria y la migración indocumentada estuvieron presentes, pero los resultados no fueron contundentes respecto al apoyo o el rechazo del electorado a candidatos a favor o en contra de medidas anti-inmigrantes o de una reforma migratoria integral. ${ }^{54}$ Estas situaciones demuestran que la ambigüedad de la opinión pública -y sus representantes en el gobierno- sobre los costos y beneficios de la migración indocumentada sigue siendo uno de los principales retos para avanzar en el desarrollo de políticas en este tema. ${ }^{55}$

Si bien estas reacciones a nivel local reflejan ineficiencias de fondo y de forma en las leyes migratorias estadounidenses, cuyas consecuencias afectan negativamente tanto a la población nativa como a los inmigrantes, también han tenido un efecto positivo al movilizar a una multiplicidad de actores que están interesados en participar de forma constructiva en la discusión del tema. Como lo demuestran los numerosos casos de demandas legales exitosas y la revocación de algunas ordenanzas, a pesar de que los grupos anti-inmigrantes generalmente tienen más recursos y dominan el discurso en este tema, las coaliciones de defensa de los derechos de los inmigrantes que se han formado y fortalecido a lo largo de los años tienen una influencia cada vez mayor en el debate y en las medidas relacionadas con la migración, al menos - por ahora- en el ámbito local..$^{56}$

contra de la migración indocumentada y del centro de contratación para jornaleros. Además, el Concejo aprobó solicitar el acuerdo para enviar a oficiales de la policía al programa de entrenamiento con las autoridades de migración y aduanas que les permitirá procesar y detener a inmigrantes indocumentados que sean identificados durante investigaciones criminales. Herndon es una de las primeras ciudades que participa en el programa (véase Bill Turque y Karin Brulliard «Police Enforcement of Immigration Laws Raises Worry Foreigners Without Documents Say They May Leave Out of Fear», The Washington Post, 1 de octubre de 2006; y «Herndon does a 180degree turn", Editorial, The Washington DC Examiner, 13 de octubre de 2006).

${ }^{54}$ Respecto a la influencia del voto latino también existen diferencias de opinión. Algunos autores argumentan que los electores latinos sí tuvieron un impacto importante en los resultados de las elecciones al rechazar a los candidatos republicanos con discursos intransigentes respecto a la migración (73\% de los latinos votaron por el Partido Demócrata y 26\% por el Partido Republicano). Aunque no se identifica como la principal ni la única razón por la que votaron en su contra (sólo 9\% de los latinos identificaron la migración como tema prioritario, mientras que dieron mayor importancia a la educación, la economía, el empleo y la guerra en Irak), analistas como Tamar Jacoby argumentan que una actitud moderada y constructiva en el tema migratorio pueda ganarle un mayor apoyo al Partido Republicano por parte este grupo de votantes, quienes tendrán un impacto cada vez mayor dadas las tendencias demográficas en el país. No obstante, cabe destacar que también hubo candidatos demócratas que ganaron la elección a pesar de promover medidas para imponer medidas restrictivas frente a la migración (véase Tamar Jacoby, «GOP Can't Lose Latinos», Los Angeles Times, 17 de noviembre de 2006 y Jorge Bustamante, «El voto latino en EU», Reforma, 14 de noviembre de 2006).

${ }_{55}$ Para una discusión más detallada sobre este tema, véase Cornelius, 2000.

${ }^{56}$ Un ejemplo interesante es el cambio de posición que ha adoptado desde el año 2000 el grupo de sindicatos AFL-CIO que pasó de ser una organización abiertamente anti-inmigrante a postularse a 
Frente al estancamiento y la lentitud que tradicionalmente ha caracterizado al debate migratorio en el Congreso, estas situaciones proporcionan evidencia concreta sobre la necesidad de responder a un fenómeno que incide en la vida diaria de la población estadounidense y ha creado nuevas presiones para las autoridades, sobre todo en pequeñas localidades en donde los efectos de la migración pueden ser más notorios e inmediatos. Aunadas a las manifestaciones multitudinarias a favor de una reforma integral que ocurrieron entre febrero y mayo de 2006, reflejan la creciente importancia del tema para grupos de la sociedad civil que normalmente no participaban en este debate.

La pregunta es si las condiciones de estas localidades y el impacto que han tenido hasta ahora en la opinión pública influye, o puede llegar a influir y cómo, en la respuesta del gobierno federal. El análisis de John Torpey (2000) sobre la descentralización del control de la frontera en las décadas recientes puede explicar algunas de las razones por las que el gobierno federal generalmente considera menos costoso en términos políticos el que el control de la migración se traslade de facto al ámbito local en lugar de buscar satisfacer a los grupos con intereses contradictorios con una reforma migratoria amplia. Según Torpey, al delegar funciones a otras instituciones y actores dentro del gobierno, el Estado puede reconciliar más fácilmente los intereses contradictorios dentro del país, calmar la ansiedad de la población, reducir los costos de la regulación y ejercer el control, incluso de forma antidemocrática, sin tener que enfrentar los costos políticos, pues no se compromete directamente con ningún grupo ni con acciones específicas.

Es posible que dada la exaltación sin precedentes que se ha dado en el ámbito local en los últimos meses, el gobierno federal estadounidense se enfrente a una presión cada vez mayor para dar una respuesta amplia al tema. Además, el hecho de que se hayan rechazado muchas de estas ordenanzas e iniciativas por medio de acciones legales significa que tampoco la vía de la legislación local será suficiente para calmar a los grupos que exigen un mayor control de la migración. Esto puede propiciar acciones más radicales que exijan una respuesta inmediata del gobierno federal. Sin embargo, existe el riesgo de que la respuesta del Congreso sea, como hasta ahora, promover medidas restrictivas de corto plazo que se enfoquen en el control de la frontera sin incluir programas de trabajadores temporales o esquemas de regularización para los cerca de 12 millones de inmigrantes indocumentados que ya se encuentran en el país. Este escenario implicaría que el problema de fondo quedaría irresuelto y las razones por las que algunos grupos están molestos o preocupados seguirán existiendo.

favor de la sindicalización de los inmigrantes indocumentados y de medidas que favorezcan su situación en el país. En agosto de 2006, la AFL-Cio se asoció con la red de organizaciones de jornaleros para promover la regularización del estatus migratorio de los trabajadores indocumentados, apoyándolos por medio de abogados y expertos en cabildeo (Karin Brulliard, «AFL-CIO Aligns With Day-Laborer Advocates», The Washington Post, 10 de agosto de 2006). 
Mientras tanto, las medidas incompletas y dispersas que se discuten (y en algunos casos han entrado en vigor) en numerosas ciudades y condados del país, seguirán atentando en contra los derechos civiles de grupos de inmigrantes. Aunque las victorias legales dan una señal positiva sobre la supremacía de la ley constitucional, las decisiones de las cortes no eliminan el ambiente anti-inmigrante, ni evitan la discriminación. Como lo describe un editorial del diario The New York Times, «no se puede imponer el sentido común desde el tribunal. ${ }^{57}$ Una tendencia que habrá que monitorear son las consecuencias de estas medidas para los dueños de pequeños negocios y empleadores -aunque hasta el momento pocos representantes de estos grupos han tomado una posición activa en el debate-y las presiones que puedan ejercer en algún momento en contra de las leyes e iniciativas -tanto a nivel local como federal- que obstaculizan la llegada de mano de obra y consumidores para sus negocios y empresas.

Frente a esta situación, los países que, como México, buscan influir de alguna manera en el rumbo que tome el debate sobre la reforma migratoria en Estados Unidos, necesariamente deben tomar en cuenta las situaciones a nivel local en el diseño de sus estrategias de cabildeo. El hecho de que existan estas confrontaciones indica que uno de los retos principales es modificar las percepciones negativas de la opinión pública norteamericana sobre los inmigrantes, empezando desde las pequeñas ciudades y poblados. En este aspecto, puede aprovecharse la existencia de un importante número de organizaciones y grupos a nivel nacional y local (organizaciones pro-inmigrantes, sindicatos, iglesias, medios de comunicación, ONG, centros de apoyo comunitarios, etc.) que reconocen las contribuciones de los inmigrantes a la sociedad y a la economía estadounidense. Estos grupos han demostrado una gran capacidad para organizarse y tomar acciones para promover medidas que favorezcan a los inmigrantes y garanticen el respeto a sus derechos humanos. Aunque no siempre logren sus objetivos, su labor deja claro que la sociedad estadounidense no es de una sola opinión respecto a cómo resolver la situación migratoria. La relevancia de estos actores también indica que enfocarse en el cabildeo a nivel del Ejecutivo o del Congreso en Washington es insuficiente. México tiene la red consular más extensa en Estados Unidos y puede aprovechar su enorme experiencia y conocimiento de las situaciones locales para desarrollar estrategias de largo alcance con el apoyo de la variedad de actores de la sociedad civil, e incluso de autoridades estatales y locales, que buscan promover una imagen positiva de la población inmigrante y defender sus derechos. Esta podría ser una buena oportunidad para influir de forma positiva en el debate migratorio iniciando desde los suburbios y las ciudades pequeñas en lugar de concentrar toda la atención y el trabajo del gobierno mexicano en lo que ocurre en las principales ciudades o en la capital.

\footnotetext{
${ }^{57}$ «Day Laborers' Rights», Editorial, The New York Times, 24 de noviembre de 2006.
} 


\section{BIBLIOGRAFÍA}

Cornelius, Wayne A. (2000), "Acogida ambivalente: economía, cultura y etnia en las políticas de inmigración de los Estados Unidos», Foro Internacional, núm. 16, pp. 41-63.

DéLAno, Alexandra, "Jornaleros (Day-Laborers)», Boletín Temático, Instituto de los Mexicanos en el Exterior, vol. 2, núm. 1, abril de 2006, disponible en http://www.ime. gob.mx/noticias/boletines_tematicos/Jornaleros.pdf.

FINE, Janice, «Worker centers -organizing communities at the edge of a dream», Economic Policy Institute, EPI Briefing Paper \#159, 14 de diciembre de 2005, (http://www.epinet.org/ briefingpapers/159/bp159.pdf).

Gordon, Jennifer (2005), Suburban Sweatshops: The Fight for Immigrant Rights, Cambridge, Mass., Belknap/Harvard University Press.

Instituto De LOS MeXICANOS en el EXTeRIOR, «Sistema de Monitoreo sobre Iniciativas Locales en Materia de Control Migratorio», México D.F., julio 2007, disponible en http:// www.ime.gob.mx/smilcm.htm

NATIONAL ImMigration ForUm, «Backgrounder: Immigration Law Enforcement by State and Local Police», National Immigration Forum, mayo de 2004, disponible en http:/ /www.immigrationforum.org/DesktopDefault.aspx?tabid=572, última consulta: 20 de noviembre de 2006.

Seghetti, Lisa M., Viña, Stephen R. y Ester, Karma, «Enforcing Immigration Law: The Role of State and Local Law Enforcement», Congressional Research Service, The LibraryofCongress, 11 demarzode2004, disponibleen (http://www.policyalmanac.org/ social_welfare/archive/immigration_enforcement.pdf, última consulta: 21 de noviembre de 2006.

ToRPeY, John (2000), «States and the Regulation of Migration in the Twentieth Century North Atlantic World», en Andreas, Peter y Timothy Snyder (editores), The Wall Around the West: State Borders and Immigration Controls in North America and Europe, Lanham, Rowman and Littlefield, pp. 31-54.

Valenzuela, Abel, Nik Theodore, Edwin Meléndezy Ana Luz Gonzalez, On the Corner: Day Labor in the United States, UCLA, enero 2006, disponible en http://www.sscnet.ucla.edu/ issr/csup/uploaded_files/Natl_DayLabor-On_the_Corner1.pdf 